\section{Jupiter's magnetosphere and aurorae observed by the Juno spacecraft during its first polar orbits}

J. E. P. Connerney, ${ }^{1,2 *}$ A. Adriani, ${ }^{3}$ F. Allegrini, ${ }^{4}$ F. Bagenal, ${ }^{5}$ S. J. Bolton, ${ }^{4}$ B. Bonfond, ${ }^{6}$ S. W. H. Cowley, ${ }^{7}$ J.-C. Gerard, ${ }^{6}$ G. R. Gladstone, ${ }^{4}$ D. Grodent, ${ }^{6}$ G. Hospodarsky, ${ }^{8}$ J. L. Jorgensen, ${ }^{9}$ W. S. Kurth, ${ }^{8}$ S. M. Levin, ${ }^{10}$ B. Mauk, ${ }^{11}$ D. J. McComas, ${ }^{12}$ A. Mura, ${ }^{3}$ C. Paranicas, ${ }^{11}$ E. J. Smith, ${ }^{10}$ R. M. Thorne, ${ }^{13}$ P. Valek, ${ }^{4}$ J. Waite ${ }^{4}$

The Juno spacecraft acquired direct observations of the jovian magnetosphere and auroral emissions from a vantage point above the poles. Juno's capture orbit spanned the jovian magnetosphere from bow shock to the planet, providing magnetic field, charged particle, and wave phenomena context for Juno's passage over the poles and traverse of Jupiter's hazardous inner radiation belts. Juno's energetic particle and plasma detectors measured electrons precipitating in the polar regions, exciting intense aurorae, observed simultaneously by the ultraviolet and infrared imaging spectrographs. Juno transited beneath the most intense parts of the radiation belts, passed about 4000 kilometers above the cloud tops at closest approach, well inside the jovian rings, and recorded the electrical signatures of high-velocity impacts with small particles as it traversed the equator.

$\mathrm{T}$ he Juno Mission serves two principal science objectives. The first is to understand the origin and evolution of Jupiter, informing the formation of our solar system and planetary systems around other stars. Servicing this objective, Juno's measurements of gravity, magnetic fields, and atmospheric composition and circulation probe deep inside Jupiter to constrain its interior structure and composition (1). The second objective takes advantage of Juno's close-in polar orbits to explore Jupiter's polar magnetosphere and intense aurorae (2). From a vantage point above the poles, Juno's fields and particles instrumentation gather direct in situ observations of the particle populations exciting the aurora, which are imaged simultaneously by Juno's ultraviolet (UV) and infrared (IR) imaging spectrographs.

Juno's payload includes a suite of fields and particle instruments for in situ sampling of Jupiter's environment. Juno's magnetometer investigation (MAG) consists of a pair of vector fluxgate magnetometers and proximate star cameras for accu-

${ }^{1}$ Space Research Corporation, Annapolis, MD 21403, USA. 2NASA Goddard Space Flight Center, Greenbelt, MD 20771, USA. ${ }^{3}$ Institute for Space Astrophysics and Planetology, National Institute for Astrophysics, Rome, 00133, Italy.

${ }^{4}$ Southwest Research Institute, San Antonio, TX 78238, USA. ${ }^{5}$ Laboratory for Atmospheric and Space Physics, University of Colorado, Boulder, C0 80303, USA. Institut d'Astrophysique et de Geophysique, Universite de Liege, Liege, B-4000 Belgium. ${ }^{7}$ University of Leicester, Leicester, LE1 7RH, UK. ${ }^{8}$ University of lowa, lowa City, IA 52242, USA. ${ }^{9}$ National Space Institute Technical University of Denmark, Kongens Lyngby, 2800, Denmark. ${ }^{10}$ Jet Propulsion Laboratory/California Institute of Technology, Pasadena, CA 91109, USA. ${ }^{11}$ Johns Hopkins University, Applied Physics Laboratory, Laurel, MD 20723, USA. ${ }^{12}$ Department of Astrophysical Sciences, Princeton University, Princeton, NJ 08544, USA. ${ }^{13}$ Department of Atmospheric and Oceanic Sciences, University of California-Los Angeles, LoS Angeles, CA 90095, USA.

*Corresponding author. Email: jack.connerney@nasa.gov rate mapping of the planetary magnetic field (3). The Jupiter Energetic Particle Detector Instrument (JEDI) measures electrons in the energy range from 30 to $800 \mathrm{keV}$ and ions from $10 \mathrm{keV}$ to $>1 \mathrm{MeV}(4)$, whereas the Jovian Auroral Distributions Experiment (JADE) measures electrons with energies of 0.1 to $100 \mathrm{keV}$ and ions from 5 to $50 \mathrm{keV}$ (5). Jovian radio and plasma waves are recorded with the Waves Instrument (Waves) which covers the spectrum from $50 \mathrm{~Hz}$ to $>40 \mathrm{MHz}$ (see the supplementary materials). Remote observations of the aurora are acquired by a long-slit ultraviolet spectrograph (UVS) counting individual UV photons ( 6 ) with wavelengths between 68 and $210 \mathrm{~nm}$ and the Jupiter Infrared Auroral Mapper (JIRAM), which performs imagery and spectrometry over a range (2 to $5 \mu \mathrm{m}$ ) of infrared wavelengths (7).

\section{Jupiter's magnetosphere}

Jupiter's dynamo generates the most intense planetary magnetic field in the solar system, with a dipole moment $\sim 20,000$ times that of Earth and surface field magnitudes ( 1 bar level; fluid planets use a pressure level for reference) about 20 times greater than Earth's (8). The supersonic solar wind is slowed well upstream of Jupiter, forming a bow shock (BS) where the ionized solar wind is abruptly decelerated and heated by the obstruction created by Jupiter's magnetic field. Downstream of the shock lies a region of turbulent flow called the magnetosheath, separated from the region within (the magnetosphere) by the magnetopause (MP). Throughout the magnetosphere, charged particle motion is guided by the magnetic fields originating within Jupiter's interior and, to a lesser extent, currents distributed throughout the magnetosphere.

Juno encountered the jovian BS just once [24, June 2016, day of year (DOY) 176], at a radial distance of $128 R_{\mathrm{J}}$ (jovian radii, $1 R_{\mathrm{J}}=$ $71,492 \mathrm{~km}$ ), on initial approach to Jupiter (see the supplementary materials). Multiple MP crossings

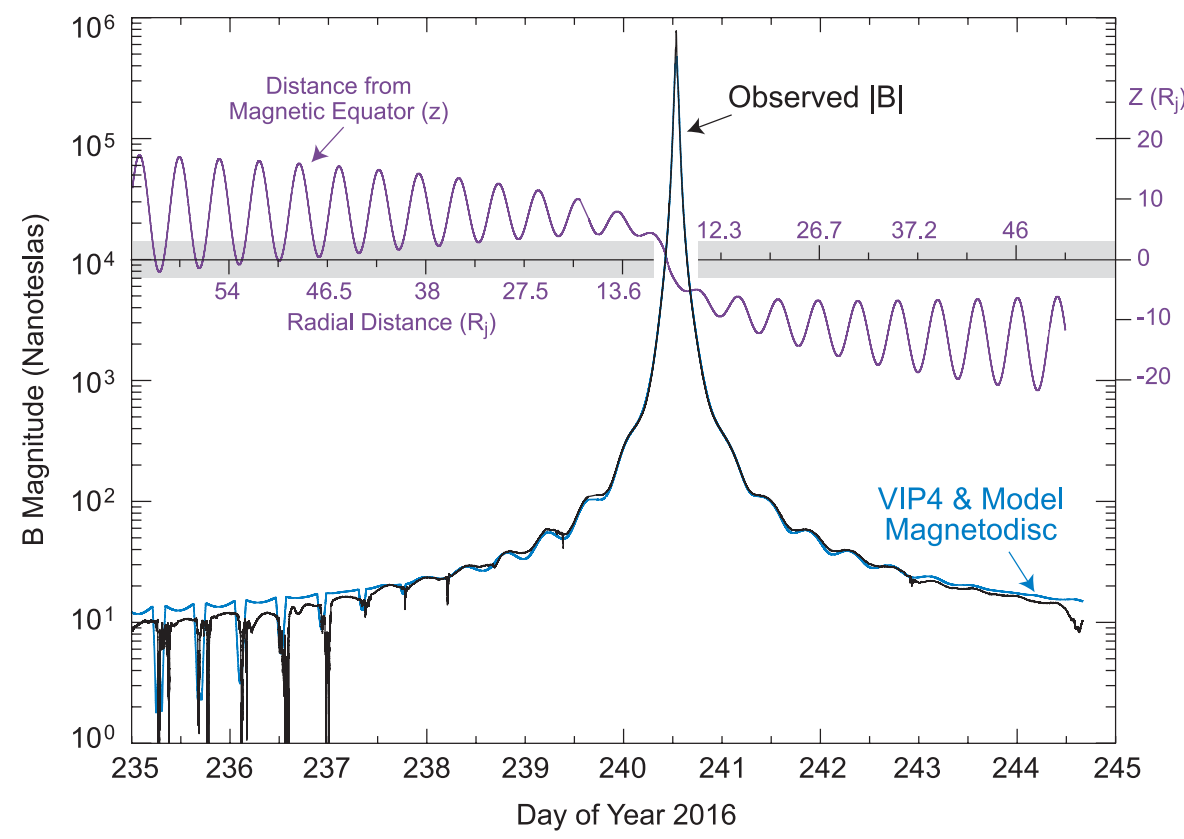

Fig. 1. Measured magnetic field magnitude (black, solid) and distance above the magnetic equator (purple) as a function of time and distance from Jupiter during Juno's first perijove pass. A model (blue) combines the planetary magnetic field [VIP4 spherical harmonic (9) model] with that of the magnetodisc (10), a system of azimuthal currents confined to within $\sim 3 R_{\mathrm{J}}$ of the magnetic equator (shaded region). The magnetic field magnitude increases by more than six orders of magnitude on approach to perijove, where a field of $7.766 \mathrm{G}$ was observed. 
were observed spanning the next 5 days (25 to 29 June, DOY 177 to 181) at radial distances of 74 to $114 R_{\mathrm{J}}$, before orbit insertion on 4 July (DOY 186). Juno's approach and first few orbits lie very nearly in the dawn meridian, so all BS and MP observations are representative of the dawn flank of Jupiter's magnetosphere. Observation of only one BS upon approach suggests that the magnetosphere was expanding in size, a conclusion bolstered by the multiple BS encounters experienced outbound (DOY 199 to 210) during the 53.5 day capture orbit at radial distances of 92 to $112 R_{\mathrm{J}}$ before apojove on DOY $213\left(\sim 113 R_{\mathrm{J}}\right)$, and at distances of 102 to $108 R_{\mathrm{J}}$ thereafter (DOY 221 to 224). Apojove during the 53.5-day orbits occurred at a radial distance of $\sim 113 R_{\mathrm{J}}$, so Juno resides at distances of $>92 R_{\mathrm{J}}$ for little more than half of its orbital period ( 29 days). Thus, on the first two orbits, Juno encountered the MP boundary a great many times at radial distances of $\sim 81$ to $113 R_{\mathrm{J}}$.

Juno's traverse through the well-ordered portion of the jovian magnetosphere is illustrated in Fig. 1, in which the measured magnetic field magnitude is compared with a magnetospheric model derived from earlier flybys. The variation in magnetic field magnitude throughout most of the 10-day interval centered on closest approach to Jupiter $\left(1.06 R_{\mathrm{J}}\right)$ at 12:53 UT is well understood based upon prior knowledge of the planetary magnetic field (8) and that of the jovian magnetodisc (10), a system of azimuthal currents flowing in a washer-shaped disc within a few $R_{\mathrm{J}}$ of the magnetic equator. Upon approach (DOY 235 to 237), precipitous decreases in the observed magnetic field magnitude result from Juno's traversal of the magnetic equator where magnetodisc currents effectively nullify the internal field. Outbound from perijove (periapsis of Jupiter orbit), Juno traveled through higher magnetic latitudes and did not penetrate the magnetodisc currents so close to Jupiter. Juno's first passage through the jovian magnetosphere, both inbound and outbound, most closely resembled the inbound portion of the high-latitude Ulysses flyby (11). These three passages are well described by a magnetodisc model with substantially less current density per unit radius (12) compared to that prevalent during the Voyager and Pioneer encounters.

The magnetic field observed in the previously unexplored region close to the planet (radius < $1.3 R_{\mathrm{J}}$ ) was dramatically different from that predicted by existing spherical harmonic models, revealing a planetary magnetic field rich in spatial variation, possibly due to a relatively large dynamo radius (1). Perhaps the most perplexing observation was one that was missing: the expected magnetic signature of intense field-aligned currents (Birkeland currents) associated with the main aurora. We did not identify large magnetic perturbations associated with Juno's traverse of field lines rooted in the main auroral oval (see the supplementary materials).

\section{Radio and plasma waves}

Juno's Waves instrument made observations of radio- and plasma-wave phenomena through-

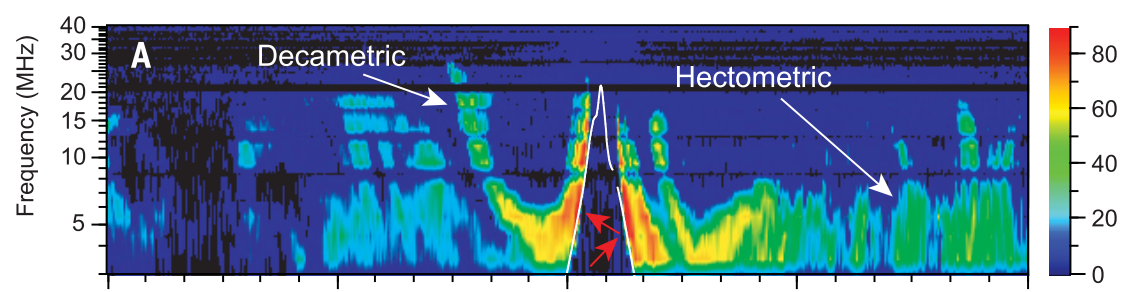

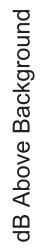
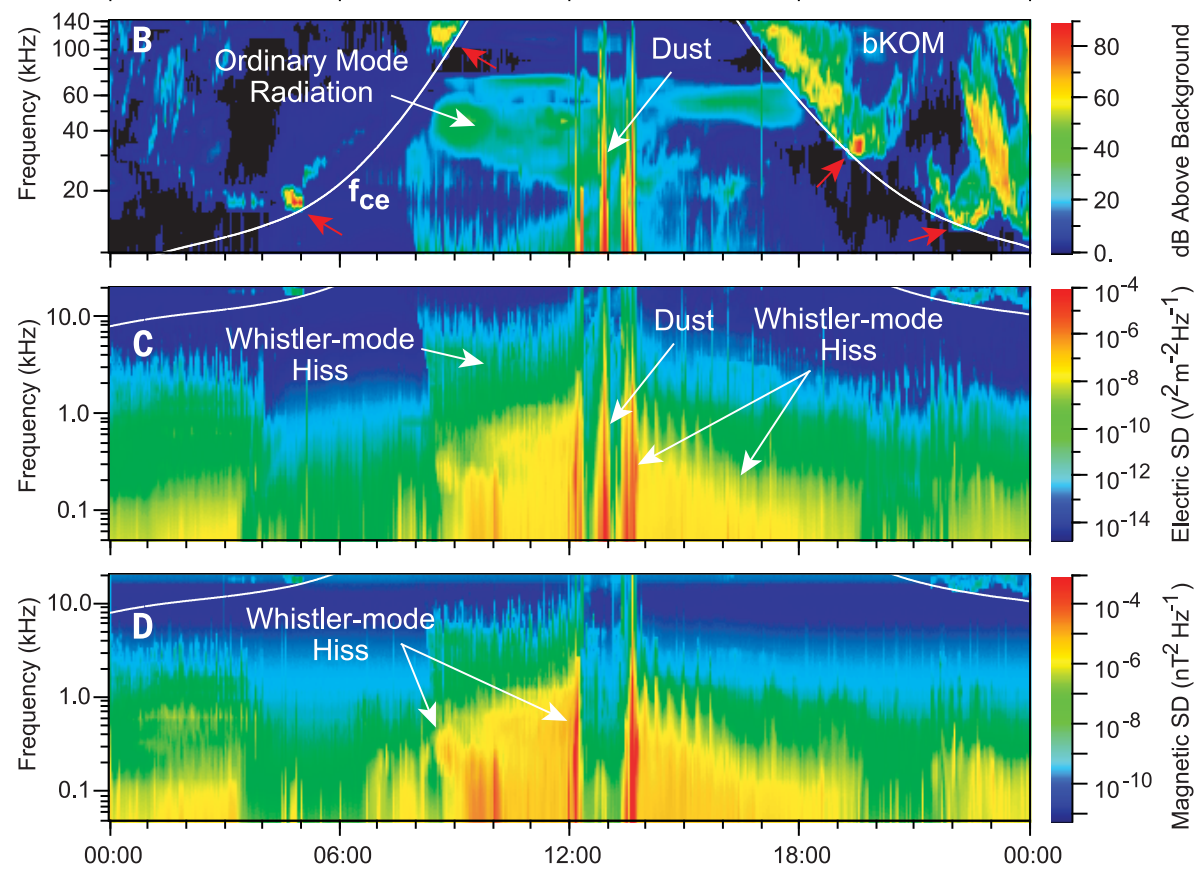

August 27, Day 240, 2016

Fig. 2. Radio- and plasma-wave observations in Jupiter's polar magnetosphere during Juno's first perijove pass. The intensity or spectral density (SD) of one electric component (A to $\mathbf{C}$ ) of waves as a function of frequency $(50 \mathrm{~Hz}$ to $40 \mathrm{MHz})$ and time and one magnetic component (D) as a function of frequency $(50 \mathrm{~Hz}$ to $20 \mathrm{kHz})$ and time is indicated by the color bar. The white curve in all panels is the electron cyclotron frequency calculated from the magnitude of the magnetic field by $f_{\text {ce }}(\mathrm{Hz})=28|\mathrm{~B}|(\mathrm{nT})$. Red arrows in $(\mathrm{A})$ and $(\mathrm{B})$ identify emissions observed with increasing intensity just above $f_{\text {ce }}$ that may indicate proximity to or passage through emission source regions.

out the first perijove (Fig. 2). These observations were obtained at low altitudes while crossing magnetic field lines mapped to Jupiter's complex auroras, so they can be compared with terrestrial observations. Jupiter's auroral radio emissions are displayed in Fig. 2A, particularly after perijove. Many of the features in the figure are organized by a fundamental mode of the plasma, the electron cyclotron frequency $f_{\text {ce }}$ [simply related to magnetic field intensity by $\left.f_{\mathrm{ce}}(\mathrm{Hz})=28|\mathrm{~B}|(\mathrm{nT})\right]$. The radio emissions in Fig. 2, A and B (and near the top of $2 \mathrm{C}$ ), are confined to frequencies at or above $f_{\text {ce. }}$. These radio emissions are thought to be generated by the cyclotron maser instability (CMI) at frequencies at or just below $f_{\text {ce }}$ (13). Because these radio emissions closely approach the line at $f_{\text {ce }}$ in several locations, and show intensification as they do so, it appears that Juno passed very close to (if not through) the source regions for these emissions.

Figure 2, C and D, shows the electric and magnetic components, respectively, of waves at frequencies below $20 \mathrm{kHz}$. The two intensifications of this emission just after 12:00 and near 13:30 UT demarcate Juno's passage over Jupiter's main auroral oval. These features are thought to be whistler-mode hiss, often called auroral hiss (14), and correspond to features in the energetic electron distributions described below. These waves exist at frequencies below the lower of $f_{\text {ce }}$ and $f_{\text {pe, }}$, the electron plasma frequency [related to electron density $n_{\mathrm{e}}$ by $n_{\mathrm{e}}=\left(f_{\mathrm{pe}} / 8980\right)^{2}$, with $n_{\mathrm{e}}$ expressed in $\mathrm{cm}^{-3}$ and $f_{\mathrm{pe}}$ in $\mathrm{Hz}$. The lack of features in the wave spectrum identifying the electron plasma frequency makes it difficult to determine the electron density. However, the identification of the emissions in Fig. 2, C and D, below a few tens of kilohertz as whistler-mode hiss and those above $20 \mathrm{kHz}$ but below $f_{\text {ce }}$ in Fig. $2 \mathrm{~B}$ as ordinary mode radio emission (perhaps related to continuum radiation) is consistent with electron densities on the order of $10 \mathrm{~cm}^{-3}$ in the polar regions. The intensifications in Fig. 2, C and D, with a period of $20 \mathrm{~min}$, after 14:00 UT, are 



Day 240 (August 27) 2016

Fig. 3. Electron and ion observations obtained during Juno's first perijove pass. A 24-hour display, centered on the Juno perijove pass, of energetic electron data from the JEDI instrument in (A and $\mathbf{B}$ ) and the lower energy JADE plasma data for ions (D) and electrons (E). The electron energy spectrogram in $(A)$ and $(E)$ sums all angular look directions, whereas the dynamic electron pitch angle spectrogram in (B) sums all instrument energy channels. The Juno spacecraft's (dipole) magnetic latitude and radial distance from Jupiter as a function of time is shown in (C). In (A), from 9:00 to 16:00 UT, the horizontal band centered on $\sim 150 \mathrm{keV}$ is an artifact caused by intense foreground electrons (energies $>700$ to $800 \mathrm{keV}$ ) that fully penetrate JEDI's electron sensor, leaving behind a fraction of their actual energy. The JADE ion spectrogram (D) sums ion counts over all directions and ion species (units of keV/charge, i.e., E/Q). suggestive of a link to pulsating auroras reported by Earth-based observers in both UV and $\mathrm{x}$-ray wavelengths.

The intense burst of noise centered near perijove in Fig. $2 \mathrm{C}$ and extending into Fig. $2 \mathrm{~B}$ is associated with dust grains impacting the Waves electric antenna (or perhaps the spacecraft). The grains impact the spacecraft with a relative velocity of $>60 \mathrm{~km} \mathrm{~s}^{-1}$, which provides sufficient kinetic energy to vaporize the grain and a portion of target material, from which a fully ionized hightemperature gas $\left(\sim 10^{5} \mathrm{~K}\right)$ evolves. The expanding plasma cloud from each impact produces an impulse in the electric channel of the Waves receiver that can be counted, yielding an estimated impact rate of a few per second near the jovigraphic equator. These grains are presumably being lost from Jupiter's ring system into the planetary atmosphere.

\section{Charged particles}

Electron and ion observations obtained during the same 24-hour interval are presented in Fig. 3.
The broad bright region between about 4:00 and 8:00 UT represents a period of time when the spacecraft dipped into Jupiter's radiation belts where high electron and ion intensities are encountered (15). This period is centered on a local minimum in (dipole) magnetic latitude, at radial distances between 7 and $10 R_{\mathrm{J}}$, and $\sim 4 R_{\mathrm{J}}$ above the magnetic equator. This is close to, but not within, the current-carrying region $\left(-3<z<3 R_{\mathrm{J}}\right.$, where $z$ is the distance from the magnetic equator) prevailing in magnetodisc models fitted to previous observations confined to lower latitudes. These observations, and intensities encountered near hours 20 to 22 UT at radial distances between 9 and $11 R_{\mathrm{J}}$, also a local minimum in magnetic latitude but now $\sim 5 R_{\mathrm{J}}$ below the magnetic equator, suggest a broader distribution of magnetodisc currents about the magnetic equator relative to more distant crossings.

At about 8:35 UT, JEDI and JADE observed localized electron intensification with JEDI electron distributions (Fig. 3B) symmetrically aligned with the local magnetic field direction, with the largest intensifications near pitch angles of $0^{\circ}$ and $180^{\circ}(16)$. This is the faint feature in Fig. $3, \mathrm{~A}$, $\mathrm{B}, \mathrm{D}$, and $\mathrm{E}$, just after the broad intensification between 4:00 and 8:00 UT. This occurred when Juno, at a distance of $\sim 6 R_{\mathrm{J}}$ from Jupiter's center, crossed magnetic field lines that map to the main northern auroral oval. The relatively dark region between $\sim 8: 40$ and $\sim 12: 00$ UT represents the traversal of the polar cap, the region poleward of the northern main auroral oval. That same dark region is repeated for the southern polar pass, between 14:00 and 18:00 UT. Within both of these polar cap regions, JEDI observed monodirectional, upward-directed electron beams confined narrowly to the measured magnetic field directions. Those beams are indicated in Fig. 3B by the brightening near $0^{\circ}$ pitch angle (8:40 to $11: 30 \mathrm{UT}$ ) and also near $180^{\circ}$ (14:00 to 18:00 UT). Beyond 18:30 UT, the spacecraft skims a region with magnetic field lines that map to the main auroral oval, where low contrast 

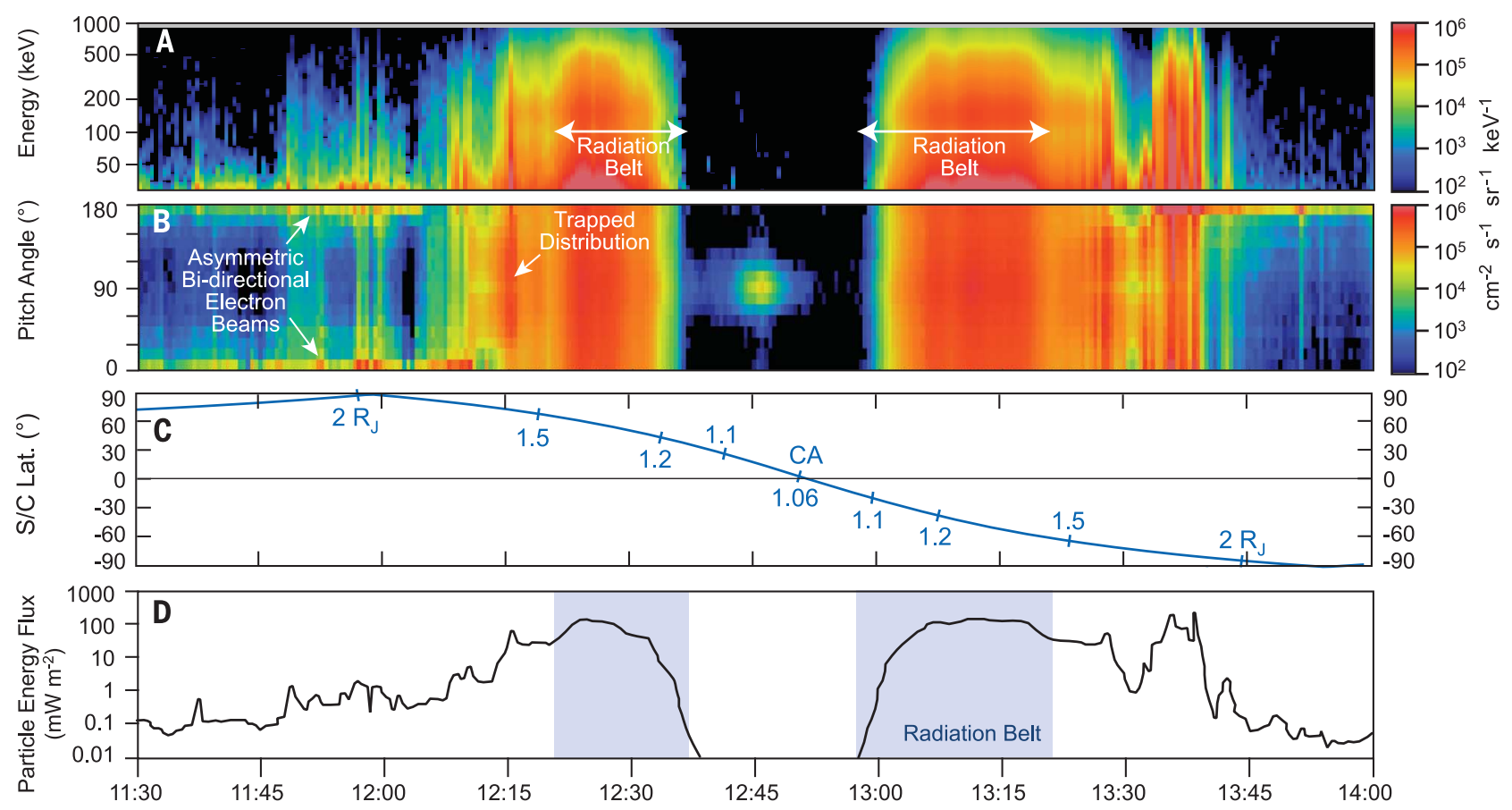

Fig. 4. Juno observations of energetic electrons and corresponding precipitating energy fluxes observed near perijove. (A and $\mathbf{B}$ ) JEDI electron energy and pitch angles observed during Juno's polar passages, showing (left) field-aligned, bidirectional but asymmetric electron beams. These panels are similar to Fig. 3, A and B, but this energy-time spectrogram (A) shows only those downward-traveling electrons with pitch angles within $15^{\circ}$ of the magnetic field. Upward-traveling electron beams are in evidence throughout both polar caps. Downward-traveling beams (near $180^{\circ}$, left, and near $0^{\circ}$, right) can deposit energy into Jupiter's atmosphere, exciting auroral emissions. (C) Spacecraft latitude and radial distance. (D) An estimate of the energy deposition from 30 to $1000 \mathrm{keV}$ downward-traveling electrons, using intensities summed over an angle within $15^{\circ}$ of the downward field line direction (excluding the radiation belts, where very high-energy electrons penetrate detector shielding).
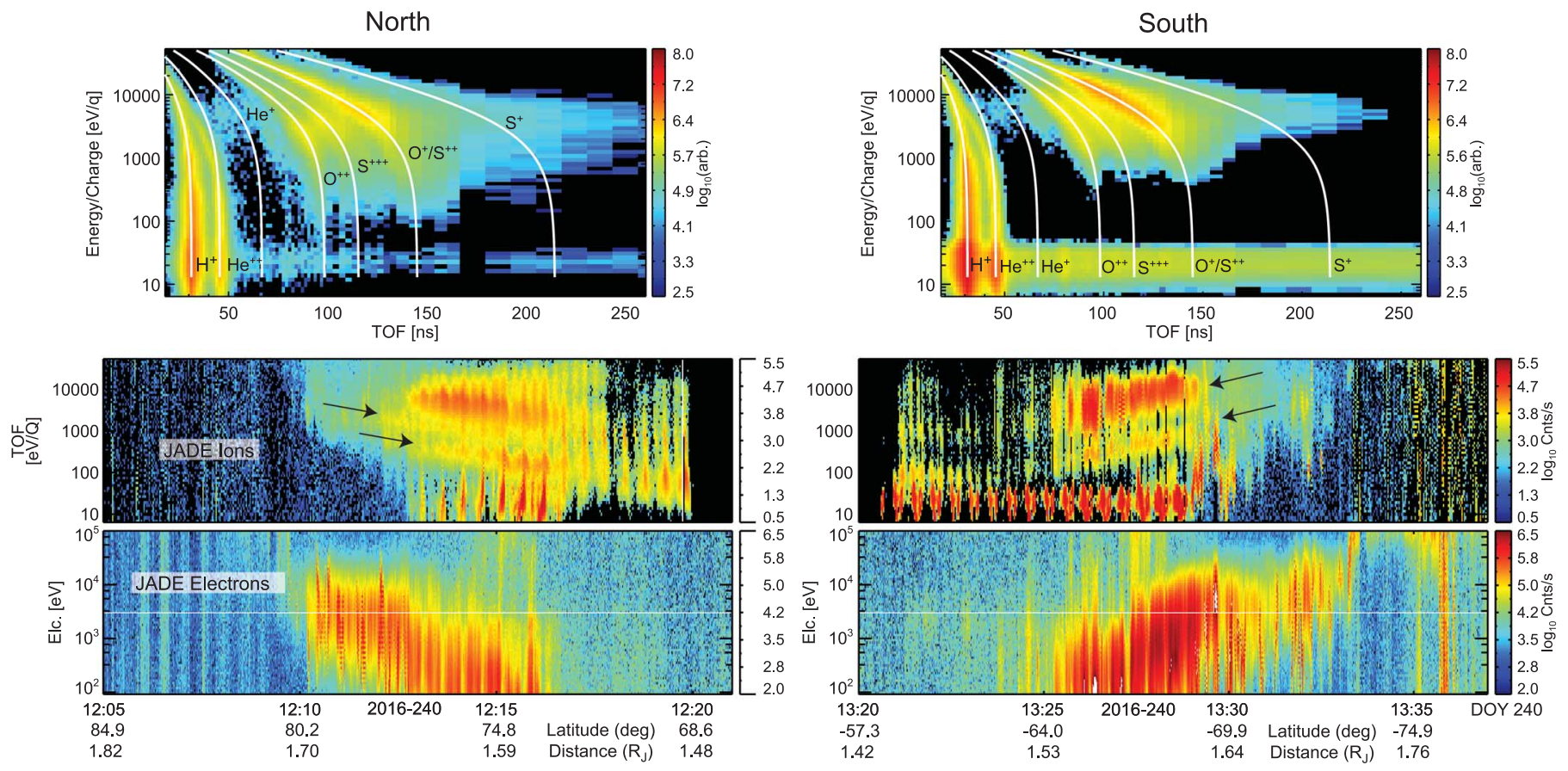

Fig. 5. Observations of plasma at latitudes equatorward of the main auroral oval in the northern (left) and southern (right) hemispheres. The top panels show the energy/TOF distributions for the time ranges below. Overlaid on the energy/TOF plots are traces showing the position of mass/ charge $(\mathrm{m} / \mathrm{q})$ of $1\left(\mathrm{H}^{+}\right), 2\left(\mathrm{H}^{+}\right.$or $\left.\mathrm{He}^{++}\right), 4\left(\mathrm{He}^{+}\right), 8\left(\mathrm{O}^{++}\right), 10.7\left(\mathrm{~S}^{+++}\right), 16\left(\mathrm{O}^{+}\right.$or $\left.\mathrm{S}^{++}\right)$, and $32\left(\mathrm{~S}^{+}\right)$. Due to interactions in the carbon foil of the TOF section, the peak at $(\mathrm{m} / \mathrm{q})=2$ is primarily due to incident protons. lons (total) as a function of energy and time are shown in the middle panels, and electrons observed by JADE are shown in the bottom panels. 
(low $\mathrm{max} / \mathrm{min}$ ) bidirectional electron beaming is observed. Near the center of Fig. 3 $(\sim 12: 00$ to 13:30 UT), ion and electron sensors responded to additional structures mapping to the main auroral oval and also intense radiation within the high-latitude horns of the radiation belts, shown at higher temporal and spatial resolution in Fig. 4. Figure 4A is an energy-time spectrogram for only those electrons moving downward toward Jupiter with pitch angles within $\pm 15^{\circ}$ of the magnetic field. All of the downward energy distributions are monotonic, without peaks in the energy distributions common to Earth auroral distributions. In this brief time span, Juno traversed field lines mapped to the northern auroral oval, the inner radiation belt, and the southern auroral oval.

Figure $4 \mathrm{~B}$ shows more clearly, on the left, the bidirectional, but asymmetric, electron beams observed by the JEDI electron detectors within the northern polar cap. The downward-traveling electron beam (near $180^{\circ}$ at left, across the northern polar cap, and near $0^{\circ}$ at right, across the southern polar cap) deposits energy into Jupiter's upper atmosphere, potentially powering jovian auroral emissions. Figure $4 \mathrm{C}$ provides an estimate of the energy deposition contributed by 30 to $1000 \mathrm{keV}$ electrons, calculated using electron intensities within $15^{\circ}$ of the downward field line (we exclude intensities within the indicated radiation belts, where very high-energy electrons penetrate detector shielding).

Energy depositions up to $\sim 80 \mathrm{~mW} \mathrm{~m}^{-2}$ are observed in the northern hemisphere and up to $\sim 200 \mathrm{~mW} \mathrm{~m}^{-2}$ in the southern hemisphere, both observed within the geometric loss cones. These energy depositions are sufficient to account for typical auroral UV luminosities at Jupiter (17). These measurements imply that Juno may have passed beneath the acceleration region powering jovian auroral emissions, at least on this occasion.

The highest JEDI energy deposition in the northern hemisphere (near 12:15 UT) was associated with a nonbeaming diffuse precipitation, as demonstrated by the character of the pitch angle distribution at that time. Although mechanisms for the generation of angular beams with monotonic energy distributions have been discussed in the context of Earth 's polar regions $(18,19)$, the context there may be different, because intense aurora are not generally associated with those beams.

At the center of Fig. 4 ( 12:45 UT), there appears a localized population of particles (ions or electrons; particle species is unknown, because the JEDI ion sensor is turned off within $10 \mathrm{~min}$ of periapsis), evidently centered about the actual magnetic equator crossing, in agreement with that computed using spherical harmonic models of the magnetic field $\left(\sim 20^{\circ}\right.$ latitude at $\sim 92^{\circ}$ system III longitude). The feature is confined in pitch angle because the loss cones are very large so close to the planet; its origin is unknown.

During the period near closest approach, the JADE ion sensor observed three distinct ion populations (Fig. 5). In the northern hemisphere at
12:13 UT, JADE observed three ion populations, each separated in energy per charge $(\mathrm{E} / \mathrm{Q})$ and having a mean energy of $\sim 5,0.2$, and $0.02 \mathrm{keV} / \mathrm{Q}$, respectively. The mean energy of the two higherenergy ion populations decreased with time until their abrupt disappearance at 12:20 UT, coinciding with the spacecraft moving equatorward of the Io (Jupiter's innermost Galilean satellite) flux tube (IFT) footprint (20). The time-of-flight (TOF) spectra (Fig. 5) show strong peaks for mass per charge $(\mathrm{m} / \mathrm{Q})$ of $1\left(\mathrm{H}^{+}\right), 8\left(\mathrm{O}^{++}\right), 10.67\left(\mathrm{~S}^{+++}\right)$, $16\left(\mathrm{O}^{+}\right.$or $\left.\mathrm{S}^{++}\right)$, and a weaker peak for $32\left(\mathrm{~S}^{+}\right)$. The strongest signal is from $\mathrm{m} / \mathrm{Q}$ of 16 . These ions have energies $>0.1 \mathrm{keV} / \mathrm{Q}$, appear to be moving together, and appear to originate from Io $(21,22)$. A third, lower-energy population of $10 \mathrm{~s}$ of $\mathrm{eV}$ protons is also observed during this time frame. These low-energy protons may be outflowing from Jupiter. During this pass, JADE did not detect a significant population of $\mathrm{H}_{3}{ }^{+}$ions. These three distinct ion distributions reappeared in the southern hemisphere starting at 13:21 UT as Juno moved poleward of the Io flux tube footprint. The energies and trends seen in these ion distributions are similar to those observed in the northern hemisphere.

The electron distributions showed similar energy dependence as the ions. Starting at 12:10 UT, JADE began to observe $\sim 1$ to $10 \mathrm{keV}$ electrons whose energy decreased to below the $0.1 \mathrm{keV}$ lower limit of the sensor by 12:16 UT as the spacecraft moved toward Jupiter's equator. A similar profile was observed in the southern hemisphere between 13:25 and 13:35 UT. These lowerenergy electrons are representative of electron distributions in Jupiter's plasma disk (not associated with UV main auroral emissions)

\section{Jupiter's aurorae}

Juno's UV and IR imaging spectrographs captured images and spectra of jovian northern and southern aurora from a unique vantage point above the poles. The northern auroral oval is offset from the rotation pole by virtue of the geometry of Jupiter's magnetic field, and most longitudes can be imaged from near-equatorial latitudes. Thus, many images of the northern aurora have been previously obtained in the IR (23) and UV $(24,25)$ using Earth-based and Earth-orbiting telescopes (e.g., the Hubble Space Telescope). The southern aurora is more closely confined to high polar latitudes and therefore less visible from near-equatorial latitudes.

Figure 6 displays complete polar maps of Jupiter's northern and southern UV polar aurorae and a polar map of Jupiter's southern aurora in the IR. The UV maps are an assemblage of slit scans across the polar region acquired with every spacecraft rotation (30 s). The UVS field of view is steered by spacecraft rotation and the orientation of the UVS scan mirror at the instrument entrance aperture. The UV brightness map is a measure of the energy flux of precipitating electrons that generate auroral emissions. The color ratio quantifies the amount of methane absorption (wavelengths $<140 \mathrm{~nm}$ ) and is a proxy for precipitating electron energy. The more energetic electrons penetrate deeper into the atmosphere before impacting an atmospheric molecule $\left(\mathrm{H}_{2}\right)$; UV photons originating deep in the atmosphere suffer more absorption (higher color ratio). Color ratios have long been observed from Earth orbit $(26,27)$, but with difficulty owing to the geometry of observation.

Jupiter's night-side UV aurora show a very intense and strongly absorbed (high color ratio) patch of outer emissions (at system III longitudes $\sim 230^{\circ}$ to $280^{\circ}$ ) in the northern polar region and a complex structure protruding from the main auroral oval into the polar region in the south (system III longitudes $\sim 20^{\circ}$ to $40^{\circ}$ ). Substantial brightness and color ratio differences are seen between the hemispheres, attributed to either hemispheric asymmetries (26) or temporal variations.

The IR intensity image of the southern aurora (Fig. 6E) is assembled from $25 \mathrm{~L}$-band filter images ( 3.3 to $3.6 \mu \mathrm{m}$ ). Intensity observed in this band is dominated by multiple $\mathrm{H}_{3}{ }^{+}$emission lines, observed with high signal-to-noise ratio against a background effectively darkened by absorption due to methane (28). This image was obtained within a span of $12 \mathrm{~min} \sim 6$ hours after perijove. The single pixel spatial resolution is $\sim 150 \mathrm{~km}$. The thickness of the main oval is quite variable along the arc. The IR aurora is thicker on the dusk side of the planet and thinner on the dawn side, as in UV. The UVS image (Fig. 6C) was acquired $\sim 5$ hours before the IR image, nearly opposite in local time from the JIRAM image in Fig. 6E.

The UV and IR auroral images both show Iogenic emissions $(29,30)$-either near the instantaneous IFT footprint or along the very long wake left behind the Io footprint-and emissions associated with the other Galilean satellites (31). The Europa wake is also present (near $120^{\circ}$ longitude) in the IR image, partially obscured by main oval emissions. JIRAM can only observe in the plane orthogonal to the spacecraft spin axis. The IR color ratio map (Fig. $6 \mathrm{~F}$ ) provides a measure of the temperature of the polar atmosphere at the altitude of the $\mathrm{H}_{3}{ }^{+}$emission. It is compiled from JIRAM spectral observations by taking the ratio of two prominent $\mathrm{H}_{3}{ }^{+}$emission lines ( 3.5 and $3.7 \mu \mathrm{m}$ ). This ratio is dependent on temperature, ranging from $\sim 1.5$ at $850 \mathrm{~K}$ to $\sim 1.83$ at $1100 \mathrm{~K}(32)$. JIRAM spectra suggest that the polar cap region interior to the main oval between longitudes $30^{\circ}$ to $90^{\circ} \mathrm{W}$ is colder than the surrounding area.

Three typical jovian auroral regions (outer, main, and inner emissions) can be identified in both the UV (25) and IR (33), together with well-identified substructures, such as the Io and Ganymede footprints (34), the large regions of UV emission associated with plasma injection signatures $(35,36)$ and the main emission discontinuity around noon. The overall aurora brightness level is somewhat dim, but not atypically so (37), and the UV color ratio values are similar to previous observations $(26,27)$. Although there is much similarity between UV and IR auroras, there are differences related to the emission 

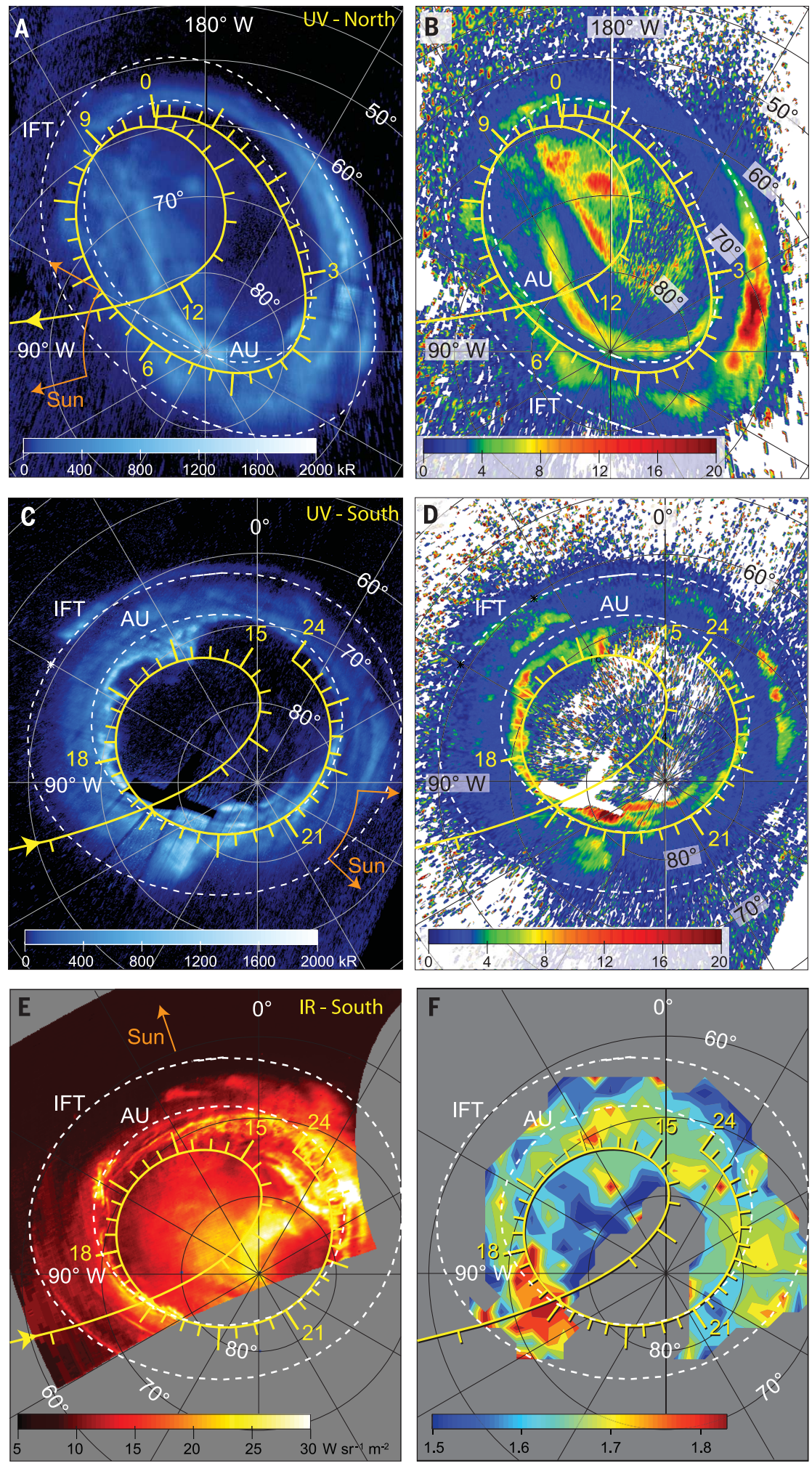

mechanisms at work in both cases. UV emissions are more directly responsive to precipitating particles and, as such, evidence rapid time variations (37). In contrast, IR emissions are thermally excited and provide a measurement of upper atmospheric temperature and thereby a measure of in its magnetosphere (39).
Fig. 6. Juno observations of jovian aurorae. Orthographic polar projections at the 1 bar level of the UV (A to $\mathbf{D}$ ) and infrared ( $\mathbf{E}$ and $\mathbf{F}$ ) auroral emissions, comparing intensities (left column) and spectral color ratios (right column) with the model auroral oval (AU, innermost dashed oval) and IFT footprint (outer dashed circle), computed using the VIP4 model internal field (9) and model magnetodisc. The footprint of the spacecraft is shown by the solid line, with hourly tic marks to facilitate comparison with in situ fields and particles observations shown in Figs. 1 to 4. Top panels are northern UV aurora; middle and bottom panels compare UV and IR southern aurora. UV intensities are summed between 60 and $180 \mathrm{~nm}$, and UV color ratios are given as 155 to $162 \mathrm{~nm}$ and 123 to $130 \mathrm{~nm}$. UVS north (south) polar data acquired between 9:52:35 and 10:40:59 (13:22:39 and 14:34:12) UT on DOY 240, 2016. The infrared aurora (E) is a mosaic of $25 L$ band filter images (3.3 to 3.6 um) obtained with 1-s integration time from between 18:09:21 and 18:22:10 UT on DOY 240, showing emission due to the $\mathrm{H}_{3}{ }^{+}$ ion. The spectral color ratio $(F)$ is obtained from JIRAM spectra acquired between 14:45 and 19:51 UT as the ratio of two $\mathrm{H}_{3}{ }^{+}$emission lines $(3.5 \mu \mathrm{m} / 3.7 \mu \mathrm{m})$ that is diagnostic of temperature (blue, relatively cold; red, hot). Gray regions are areas devoid of data.

\section{Summary}

Juno has provided observations of fields and particles in the polar magnetosphere of Jupiter, as well as high-resolution images of the auroras at UV and IR wavelengths. Although many of the observations have terrestrial analogs, it appears that different processes are at work in exciting the aurora and in communicating the ionospheremagnetosphere interaction. We observed plasmas upwelling from the ionosphere, providing a mechanism whereby Jupiter helps populate its magnetosphere. The weakness of the magnetic field-aligned electric currents associated with the main aurora and the broadly distributed nature of electron beaming in the polar caps suggest a radically different conceptual model of Jupiter's interaction with its space environment. The (precipitating) energetic particles associated with jovian aurora are very different from the peaked energy distributions that power the most intense auroral emissions at Earth.

\section{REFERENCES AND NOTES}

energy deposition, whether by particle precipitation or Joule heating. The IR aurora responds more slowly to energy input $(28,38)$ and is diagnostic of the transfer of angular momentum from Jupiter to (ionized) mass flowing outward
1. S. J. Bolton et al., Science 356, 821-825 (2017).

2. F. Bagenal et al., Space Sci. Rev. 2014, 1-69 (2014)

3. J. E. P. Connerney et al., Space Sci. Rev. 10.1007/s11214-017-0334-z (2017).

4. B. H. Mauk et al., Space Sci. Rev. 10.1007/s11214-013-0025-3 (2013)

5. D. J. McComas et al., Space Sci. Rev. 10.1007/s11214-013-9990-9 (2013) 
6. G. R. Gladstone et al., Space Sci. Rev. 10.1007/s11214-014-0040-z (2014).

7. A. Adriani et al., Space Sci. Rev. 10.1007/s11214-014-0094-y (2014).

8. J. E. P. Connerney, in Treatise in Geophysics, G. Schubert, T. Spohn, Eds., vol. 10.06 (Elsevier, Oxford, UK, 2015), pp. 195-237.

9. J. E. P. Connerney, M. H. Acuña, N. F. Ness, T. Satoh, J. Geophys. Res. 103, 11,929-11,939 (1998).

10. J. E. P. Connerney, M. H. Acuña, N. F. Ness, J. Geophys. Res. 86, 8370-8384 (1981)

11. A. Balogh et al., Science 257, 1515-1518 (1992)

12. The Pioneer 10, Voyager 1 , Voyager 2, and outbound leg of the Ulysses flyby were well modeled within $30 R_{\lrcorner}$of Jupiter, using the magnetodisc model cited in (10) with a current disc in the magnetic equator having an inner and outer radius of 5 and $50 R_{\mathrm{J}}$, respectively; a thickness of \pm 2.5 or $3 R_{\mathrm{j}}$; and a current constant of 225. The Ulysses inbound trajectory and Juno's first perijove both required substantially less current ( $45 \%$ less) and a larger outer radius of the currentcarrying magnetodisc $\left(90 R_{\mathrm{J}}\right)$

13. R. A. Treumann, Astron. Astrophys. Rev. 13, 229-315 (2006).

14. D. A. Gurnett, J. Geophys. Res. 71, 5599-5615 (1966).

15. M. de Soria-Santacruz et al., J. Geophys. Res. 121, 9732-9743 (2016).

16. Charged particles travel in helical trajectories about the magnetic field. The angle between a particle's velocity vector and the magnetic field direction is referred to as the "pitch angle," which, in a nonuniform magnetic field, varies with the ratio between the perpendicular and parallel components of the particle's velocity. This important quantity determines whether a particle will bounce back and forth, trapped in a nonuniform magnetic field, or, for sufficiently small pitch angles, travel far enough to be absorbed in the atmosphere.

17. J. T. Clarke, in Auroral Phenomenology and Magnetospheric Processes: Earth And Other Planets, A. Keiling, E. Donovan, F. Bagenal, T. Karlsson, Eds. (American Geophysical Union, Washington, DC, 2012), pp. 113-122.

18. C. W. Carlson et al., Geophys. Res. Lett. 25, 2017-2020 (1998).

19. B. H. Mauk, J. Saur, J. Geophys. Res. 112, A10221 (2007)
20. The IFT footprint is the area in the ionosphere that maps along magnetic field lines to the satellite lo, orbiting in the Jovigraphic equator at $\sim 5.9 R_{\lrcorner}$radial distance. It is sometimes referred to the "instantaneous" IFT footprint, to distinguish it from the oval traced out in the polar region by the locus of IFT footprints generated as lo moves about Jupiter in system III longitude. The "extended" IFT footprint, or "tail," is the trailing portion of the (instantaneous) footprint

21. I. Kupo, Y. Mekler, A. Eviatar, Astrophys. J. 205, L51-L53 (1976).

22. N. Thomas, F. Bagenal, T. W. Hill, J. K. Wilson, in Jupiter: The Planet, Satellites and Magnetosphere, F. Bagenal, T. Dowling, W. B. McKinnon, Eds., vol. 561 (Cambridge Univ. Press, Cambridge, UK, 2004), pp. 561-591.

23. T. Stallard, S. Miller, H. Melin, in Auroral Phenomenology and Magnetospheric Processes: Earth and Other Planets, A. Keiling, E. Donovan, F. Bagenal, T. Karlsson, Eds., Geophys. Mono. Series, vol. 197 (American Geophysical Union, Washington, DC, 2012), pp. 215-224.

24. J. T. Clarke et al., in Jupiter: The Planet, Satellites, and Magnetosphere, F. Bagenal, T. E. Dowling, W. B. McKinnon, Eds. (Cambridge Univ. Press, Cambridge, UK, 2005), pp. 639-670.

25. D. Grodent, Space Sci. Rev. 187, 23-50 (2015).

26. J.-C. Gérard, D. Grodent, A. Radioti, B. Bonfond, J. T. Clarke, Icarus 226, 1559-1567 (2013).

27. J. Gustin et al., Icarus 268, 215-241 (2016)

28. J. E. P. Connerney, T. Satoh, Philos. Trans. R. Soc. Lond. A 358 , 2471-2483 (2000).

29. J. E. P. Connerney, R. Baron, T. Satoh, T. Owen, Science 262 1035-1038 (1993)

30. J. T. Clarke et al., Science 274, 404-409 (1996).

31. J. T. Clarke et al., Nature 415, 997-1000 (2002).

32. B. M. Dinelli, C. R. Le Sueur, J. Tennyson, R. D. Amos, Chem. Phys. Lett. 232, 295-300 (1995).

33. T. Satoh, J. E. P. Connerney, Icarus 141, 236-252 (1999)

34. B. Bonfond, in Auroral Phenomenology and Magnetospheric Processes: Earth And Other Planets, A. Keiling, E. Donovan,

F. Bagenal, T. Karlsson, Eds. (American Geophysical Union, Washington, DC, 2012) pp. 133-140.

35. B. H. Mauk et al., Nature 415, 1003-1005 (2002).
36. B. Bonfond et al., Geophys. Res. Lett. 39, L01105 (2012).

37. J. T. Clarke et al., J. Geophys. Res. 114, A05210 (2009).

38. R. Baron, T. Owen, J. E. P. Connerney, T. Satoh, J. Harrington, Icarus 120, 437-442 (1996)

39. T. W. Hill, J. Geophys. Res. 84, 6554-6558 (1979).

\section{ACKNOWLEDGMENTS}

We thank project and support staff at the Jet Propulsion Laboratory (JPL), Lockheed Martin, and the Southwest Research Institute (SWRI) for the design, implementation, and operation of the Juno spacecraft. We also thank staff at Goddard Space Flight Center, the Applied Physics Laboratory, the University of lowa, and the Technical University of Denmark for providing science instruments and support. We thank the Italian Space Agency (ASI), B. M. Dinelli, and F. Fabiano for support of JIRAM The Belgian contribution to UVS is enabled via support from the Belgian Science Policy Office (BELSPO) and the European Space Agency's Program de Development d'Experiences Scientifiques (PRODEX). JPL manages the Juno mission for the principal investigator, S. Bolton, of SWRI. Supported by the Juno Project under NASA grant NNM06AAa75C to Southwest Research Institute; NASA grant NNN12AA01C to the JPL/California Institute of Technology; and Agenzia Spaziale Italiana (ASI) (A.A. and A.M.). The Juno mission is part of the New Frontiers Program managed at NASA's Marshall Space Flight Center in Huntsville, Alabama. S.W.H.C. acknowledges funding from the Science and Technology Facilities Council. As agreed with NASA, fully calibrated Juno data are released on a schedule via the NASA Planetary Data System at https://pds.nasa.gov/.

\section{SUPPLEMENTARY MATERIALS}

www.sciencemag.org/content/356/6340/826/suppl/DC1 Materials and Methods

Supplementary Text

Figs. S1 to S4

References (40-42)

13 December 2016; accepted 20 April 2017

10.1126/science.aam5928 


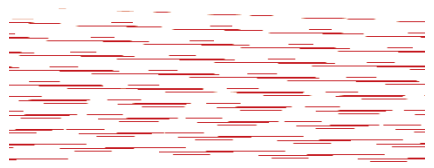

Editor's Summary
Jupiter's magnetosphere and aurorae observed by the Juno spacecraft during its first polar orbits

J. E. P. Connerney, A. Adriani, F. Allegrini, F. Bagenal, S. J. Bolton, B. Bonfond, S. W. H. Cowley, J.-C. Gerard, G. R.

Gladstone, D. Grodent, G. Hospodarsky, J. L. Jorgensen, W. S.

Kurth, S. M. Levin, B. Mauk, D. J. McComas, A. Mura, C.

Paranicas, E. J. Smith, R. M. Thorne, P. Valek and J. Waite (May $25,2017)$

Science 356 (6340), 826-832. [doi: 10.1126/science.aam5928]

\section{Juno swoops around giant Jupiter}

Jupiter is the largest and most massive planet in our solar system. NASA's Juno spacecraft arrived at Jupiter on 4 July 2016 and made its first close pass on 27 August 2016. Bolton et al. present results from Juno's flight just above the cloud tops, including images of weather in the polar regions and measurements of the magnetic and gravitational fields. Juno also used microwaves to peer below the visible surface, spotting gas welling up from the deep interior. Connerney et al. measured Jupiter's aurorae and plasma environment, both as Juno approached the planet and during its first close orbit. Science, this issue p. 821 , p. 826

This copy is for your personal, non-commercial use only.

Article Tools Visit the online version of this article to access the personalization and article tools:

http://science.sciencemag.org/content/356/6340/826

Permissions Obtain information about reproducing this article: http://www.sciencemag.org/about/permissions.dtl

Science (print ISSN 0036-8075; online ISSN 1095-9203) is published weekly, except the last week in December, by the American Association for the Advancement of Science, 1200 New York Avenue NW, Washington, DC 20005. Copyright 2016 by the American Association for the Advancement of Science; all rights reserved. The title Science is a registered trademark of AAAS. 